\title{
TopoLens: Building a CyberGIS Community Data Service for Enhancing the Usability of High-resolution National Topographic Datasets
}

\author{
$\mathrm{Hao} \mathrm{Hu}^{1}$ \\ haohu3@illinois.edu \\ Xingchen Hong ${ }^{2}$ \\ hong114@illinois.edu \\ Jeff Terstriep ${ }^{2}$ \\ Michael P. Finn ${ }^{3}$ \\ Yan Y. Liu ${ }^{2}$ \\ yanliu@illinois.edu \\ mfinn@usgs.gov \\ jefft@illinois.edu \\ Johnathan Rush ${ }^{2}$ \\ jfr@illinois.edu \\ Jeffrey Wendel ${ }^{4}$ \\ jwendel@usgs.gov \\ Shaowen Wang ${ }^{1,2}$ \\ shaowen@illinois.edu \\ ${ }^{1}$ Department of Geography and Geographic Information Science \\ University of Illinois at Urbana-Champaign \\ Champaign, IL 61820 \\ ${ }^{2}$ National Center for Supercomputing Applications \\ University of Illinois at Urbana-Champaign \\ Urbana, IL 61801 \\ ${ }^{3}$ U.S. Geological Survey \\ Center of Excellence for Geospatial Information Science \\ Denver, Colorado, USA 80225 \\ ${ }^{4}$ U.S. Geological Survey \\ Center of Excellence for Geospatial Information Science \\ Rolla, Missouri, USA 65401
}

\begin{abstract}
Geospatial data, often embedded with geographic references, are important to many application and science domains, and represent a major type of big data. The increased volume and diversity of geospatial data have caused serious usability issues for researchers in various scientific domains, which call for innovative cyberGIS solutions. To address these issues, this paper describes a cyberGIS community data service framework to facilitate geospatial big data access, processing, and sharing based on a hybrid supercomputer architecture. Through the collaboration between the CyberGIS Center at the University of Illinois at Urbana-Champaign (UIUC) and the U.S. Geological Survey (USGS), a community data service for accessing, customizing, and sharing digital elevation model (DEM) and its derived datasets from the 10-meter national elevation dataset, namely TopoLens, is created to demonstrate the workflow integration of geospatial big data sources, computation, analysis needed for customizing the original dataset for end user needs, and a friendly online user environment. TopoLens provides online access to precomputed and on-

ACM acknowledges that this contribution was authored or co-authored by an employee, or contractor of the national government. As such, the Government retains a nonexclusive, royalty-free right to publish or reproduce this article, or to allow others to do so, for Government purposes only. Permission to make digital or hard copies for personal or classroom use is granted. Copies must bear this notice and the full citation on the first page. Copyrights for components of this work owned by others than ACM must be honored. To copy otherwise, distribute, republish, or post, requires prior specific permission and/or a fee. Request permissions from permissions@acm.org. XSEDE16, July 17-21, 2016,

(c) 2016 ACM. ISBN 978-1-4503-4755-6/16/07 . \$ \$15.00

DOI: http://dx.doi.org/10.1145/2949550.2949652
\end{abstract}

demand computed high-resolution elevation data by exploiting the ROGER supercomputer. The usability of this prototype service has been acknowledged in community evaluation.

\section{CCS Concepts}

-Information systems $\rightarrow$ Geographic information systems; •Computer systems organization $\rightarrow$ Distributed architectures; • World Wide Web $\rightarrow$ Web services;

\section{Keywords}

CyberGIS, geospatial big data, data sharing, elevation data, microservices, web-based gateway environment

\section{INTRODUCTION}

With the advances in data acquisition technologies and the popularity of location-based sensors and devices (e.g., environmental sensors, remote sensing satellites, and smart phones), data with geographic information (geospatial data) has substantially contributed to the era of "big data" in the geosciences. The increased volume and diversity of geospatial data brings unprecedented usability issues to researchers from various scientific domains such as the requirement for scalable data platforms [1, 2, 3]. Raster data, as one of the basic geospatial data types, consist of a matrix of cells organized into rows and columns and are often used to represent thematic (e.g., land-use, land-cover) or continuous (e.g., digital elevation model (DEM), satellite/aerial image) geographic information at the cell location [4]. Raster data 
become big when large study area is covered with fine spatial resolution (cell size). There are several key usability challenges for big geospatial raster data access, processing, and sharing.

Access. The procedure for big geospatial raster data access is often complicated. Various geospatial portals and geospatial clearinghouses sponsored by federal, state, regional, and local governments and agencies make geographic data more accessible to the public. For example, the U.S. Geological Survey (USGS) 3D Elevation Data Program (3DEP) distributes the National Elevation Dataset (NED) as separate image tiles through The National Map Viewer $[5,6]$. However, downloading elevation data for a study area that covers more than one tile involves downloading tiles from several URL sources. Each of these separate downloads requires manual attention not only on clicking, but watching for server ready status. A similar tedious user experience can be found on other geospatial data service portals.

Processing. This issue arises because geospatial data agencies usually distribute their data with a single specification. For example, USGS's 3DEP has a 1/3-arc-second resolution and is organized as 1x1 degree tiles with the geographic coordinate system North American Datum of 1983 (NAD83). This is roughly 10 meters per cell in the north-south dimension.The National Land Cover Dataset (NLCD), released by the Multi-Resolution Land Characteristics (MRLC) consortium (http://mrlc.gov), is distributed as one single raster in 30-meter spatial resolution and is mapped in the Albers Conic Equal Area projection. Therefore, users often have to go through several ad-hoc processing steps, such as mosaicing and clipping the data into an area of interest (AOI), transforming to a specific map projection, and resampling to consistent spatial resolution in order to prepare the data to be consumed by other analytic workflows. Those operations require users to have basic geospatial data processing knowledge and possess related geographic information system (GIS) tools. Moreover, as data resolution becomes finer, the aforementioned operations require much more computing power than what a desktop or data server can provide. In a geospatial big data environment, serving such dynamic data requests from end users must be coupled with matching computing power. CyberGIS [7], cyberinfrastructure-based GIS, can provide a holistic solution to the integration of data, computation, and online data services for the data user community.

Sharing. The community sharing of geospatial raster data usually requires additional administrative efforts including the detailed description of the earlier processing steps describing the transformation of the base data. Given that data sharing can reduce the duplication of data preparation efforts, researchers exploit community data management systems such as GeoShare [8], HydroShare [9], or commercial online GIS, e.g., ESRI ArcGIS Online (http://arcgis. com/home/), to improve research efficiency and productivity. However, fewer efforts have been made to couple the data sharing portals with a computational environment that allows on-demand, user-defined transformations of the desired data.

This paper provides a description of geospatial data and service portal developed using a cyber framework for geospatial raster data preparation and sharing. The design of the portal takes advantage of the hybrid nature of the ROGER (Resourcing Open Geospatial Education and Research) su- percomputer [10] at the CyberGIS Center and the National Center for Supercomputing Applications (NCSA). ROGER supports both cloud-based computing using OpenStack and traditional high performance computing (HPC) batch environments. The framework seamlessly integrates a web-based user environment, cloud-based services, and HPC computing resources in an asynchronous fashion.

\section{RELATED WORK}

In the United States, the Federal Geographic Data Committee (FGDC) coordinates the implementation of the $\mathrm{Na}$ tional Spatial Data Infrastructure (NSDI) with the vision of promoting the sharing of geospatial data throughout all levels of government, the private and nonprofit sectors, and the academic community [11]. To make geographic data more accessible to the public and increase the benefits of using available data, various geospatial data portals and clearinghouses sponsored by federal, state, regional, and local governments and agencies have emerged, such as Data,giv (https://data.gov) and Open Data Policy (https://whitehouse. gov/sites/default/files/omb/memoranda/2013/m-13-13.pdf). As a major contribution to the NSDI, the USGS's The $\mathrm{Na}$ tional Map program [5] provides various geographic information such as orthoimagery, elevation, hydrography, transportation, and land cover to the geospatial community. In addition to government agencies, research institutions are working collaboratively on building data acquisition, computing and sharing systems. OpenTopography [12], for example, is a geospatial data portal providing online access to high-resolution topography data through cyberinfrastructure developed at the San Diego Supercomputer Center. HydroShare [9] and GeoShare [8] are two other representative examples of collaborative environment for geospatial data and models sharing for scientists in hydrology (HydroShare) and the agricultural and environmental science domain (GeoShare).

Given the high computing capability in traditional HPC and in the on-demand resource/service provisioning feature from cloud computing, hybrid HPC has been applied as a new trend to solve various scientific problems [13, 14]. Hybrid architecture design that integrates Hadoop, scalable database, traditional HPC, and cloud has become a new trend in advanced cyberinfrastructures and can be found in XSEDE resources such as Wrangler (https://tacc.utexas. edu/systems/wrangler), JetStream(http://jetstream-cloud. org/), Bridges (http://psc.edu/bridges) to serve those who have not been users of traditional HPC systems. In the geospatial realm, researchers have discussed the opportunities and challenges for the application of cloud computing [15]. For example, a cloud-based architectural framework for data acquisition and processing services based on open geospatial standards has been developed to deal with the satellite images and applications in the area of remote sensing [16]. In geospatial industry, ESRI ArcGIS Online has been serving as a complete cloud-based platform for spatial analysis and map sharing for years. Other companies like GISCloud.com are looking at opportunities to provide web-based solutions in a Software as a Service (SaaS) fashion. Therefore, the combination of cloud-based geospatial data services (e.g., deploying on-demand services such as mapping services) and traditional HPC based geospatial data processing, leads to an ideal hybrid framework for building community data service for accessing, customizing, 
and sharing geospatial big data.

Taking advantage of advanced cyberinfrastructure, a new generation of GIS, namely cyberGIS, has emerged and developed both theoretical and technical frameworks for computing and data-intensive scientific discoveries in geospatial domains [7]. The CyberGIS Gateway [17], as one of the major modalities of CyberGIS, provides a leading online GIS environment enabled by advanced cyberinfrastructure, making it an ideal place to deploy the TopoLens prototype. Last year, a new cyberGIS supercomputer, ROGER, was deployed for geospatial problem solving. Leveraging the hybrid HPC design, ROGER provides a desirable environment for developing technical solutions for geospatial big data integration and sharing.

\section{SYSTEM DESIGN}

TopoLens has been developed using two emerging internet technologies: the Meteor JavaScript web framework (https: $/ /$ meteor.com) and Microservices Architecture. Meteor is a rapid-development environment built on Node.js. Meteor integrates with MongoDB (https://www.mongodb.com) and uses a publish-subscribe pattern to automatically propagate data changes to clients, making it ideally suited to the development of responsive single page applications (SPA).

Microservices is a way of breaking a large complex application into loosely coupled, independent modules which communicate using simple APIs. The ROGER supercomputer provides the ideal environment for developing microservice applications: control services can be deployed in the OpenStack cloud computing environment, while the big data handling can use the traditional HPC environment. The Microservices Architecture provides several benefits including:

- Re-usability - because each module implements a single task and can be accessed using a simple API, it naturally provides components that can be connected in different ways to solve different problems.

- Scalability - modules can be replicated and run on multiple servers to increase performance. Cloud environments allow the number of instances of a specific module to be quickly changed to meet changing demand.

- Fault Isolation - because modules can be replicated and put into service quickly, failure of any given module may not have serious consequences for the larger application.

- Flexibility - the well-defined module boundaries allow modules to be replaced with new technology stacks and eliminate long-term commitments to a single technology stack.

- Agile Development - the smaller, more focused modules are easier to understand for new developers, allowing them to make improvements more easily and with less debugging.

A TopoLens service diagram is shown in Figure 1. In addition to the Meteor web service, microservices include a job handler, a unified logger, web mapping and data download. The microservices interact asynchronously using a message broker to route messages within the system. The microservices act as support structures for the TopoLens batch job,

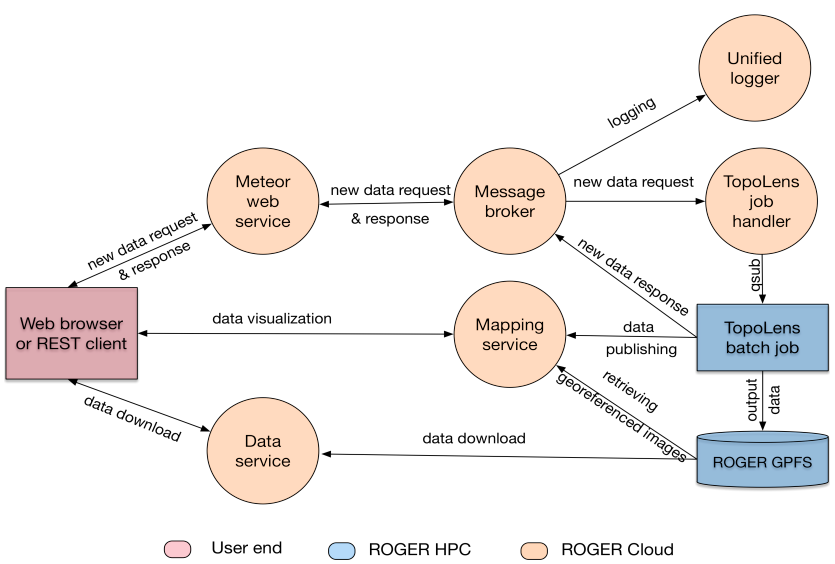

Figure 1: TopoLens Service Architecture.

which performs the necessary data transformations. Not shown in Figure 1, but equally important, is the process control service that ensures that all the other services are running.

A prototypical job flow will begin with a user request made to the Meteor web service using either the web-based interface or REST endpoints. If new data are required, the $\mathrm{Me}$ teor web service will send a request message to the message broker where the TopoLens job handler has subscribed to request messages. The handler processes the request and submits a job to the ROGER batch environment. Then, the batch job performs the desired data transformation and publishes a results message which is routed back to Meteor web service. At any point in time, a service may generate log or error messages that are handled asynchronously by the unified logger.

\subsection{TopoLens Components}

\subsubsection{Meteor Web Service}

TopoLens provides two sets of public interfaces, a Gateway web application for user access and a set of REST endpoints for programmatic access.

At the primary user interface, the TopoLens webapp uses Material Design Lite (MDL) to build a highly responsive UI that works on both personal computers and handheld touch devices. The visualization is implemented with OpenLayers (http://openlayers.org/) and Proj4Js (http://proj4js.org/) to support a great amount of different map projections (Figure 2). For third-party software or services, the set of REST endpoints can be used to perform the same tasks as those supported by the web UI by directly operating on the data products information in the database.

\subsubsection{Job Handler}

The job handler is a custom service that accepts request messages sent from the message broker. Upon message receipt, the job handler verifies the version and integrity of the request message and generates a custom batch script based on the message parameters. The batch script is submitted using an ssh command and a community account on the HPC system. The job handler integrates the generation of multiple raster products into a single batch request. The integration allows the queue wait times to be amortized 


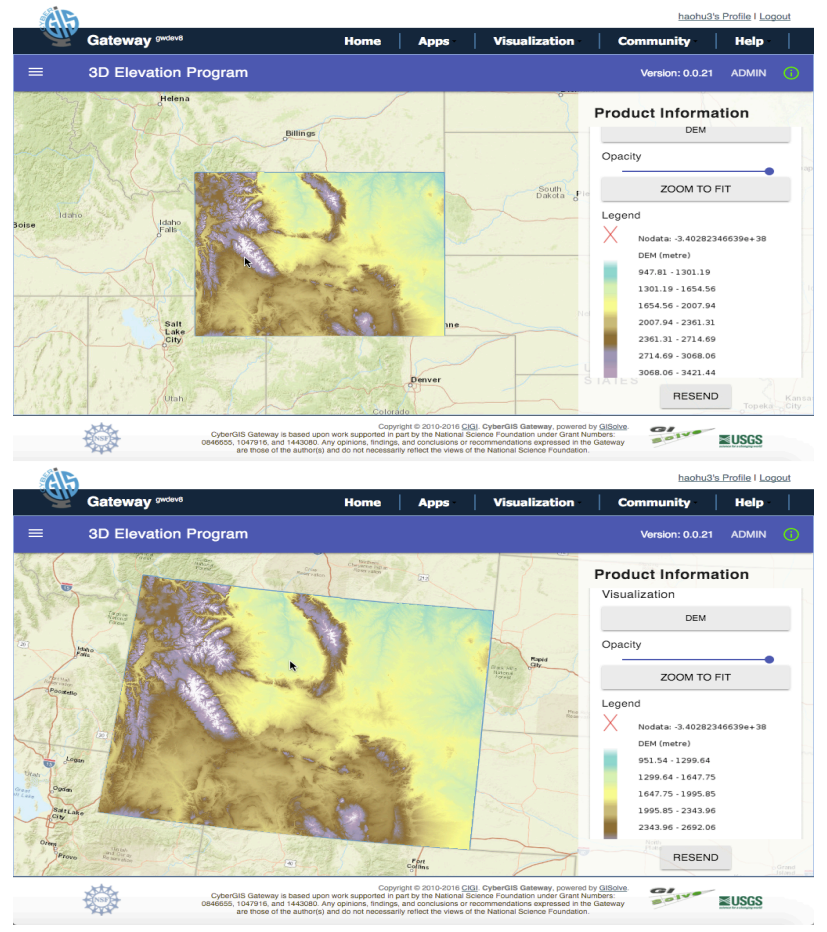

Figure 2: Same data product (elevation) in different projections (top: WGS84 Web Mercator projection, bottom: NAD83 / Conus Albers projection).

over all the requested products with only a slight increase in complexity.

Following a successful job submission, the job handler immediately returns to the listening state and no further actions are needed by the handler to complete the service. The stateless nature of the job handler allows it to be robust and provide high throughput.

\subsubsection{Batch Job}

The batch job performs the processing necessary to produce the raster products requested by the user. The TopoLens batch job assumes that all the necessary software is preinstalled and the source elevation data is pre-staged to a known location on the HPC resource. An advantage of the ROGER system is that it is designed to host large geospatial datasets and that geospatial software is maintained by its administrators. Once execution begins, the TopoLens job communicates with the TopoLens services by sending log and progress messages as well as the final response message at job completion.

To integrate with the microservice architecture, new products are written to a shared file system and their locations are included in the response message. The use of a shared file system for data passing is uncommon in microservice architecture but, by leveraging the unique capabilities of the ROGER computing environment, TopoLens is able to greatly reduce data copies and improve overall performance.

\subsubsection{Unified Logger}

One of the challenges of the microservice architecture is detecting errors and correlating errors with originating events. In the case of TopoLens, the originating event is the request message produced by the TopoLens Job Proxy. Rather than being written to local independent log files on multiple systems, log messages are sent to the message queue where a unified logger service receives and collates the messages into a single log. Each log message includes the unique request ID provided by the request message to simplify monitoring and debugging.

\subsubsection{Message Broker}

TopoLens is built using RabbitMQ (https://rabbitmq.com/), one of the most popular open source message brokers available to developers. The message broker aids in developing applications such as TopoLens by creating a buffer between the asynchronous services which may operate on different time scales.

Message brokers also support complex routing. TopoLens leverages the publication-subscribe (pub-sub) model to simplify the overall design and enhance extensibility. In the pub-sub model, each message is routed based on a topic string. Services may subscribe to specific messages based on pattern matching performed on the topic string. The unified logger listens for any message whose topic starts with "logging" and writes these to a centralized log file.

\subsubsection{Web Mapping Services}

To consume geospatial data as georeference map images over the web, map servers such as GeoServer or ArcGIS Server need to be employed to publish mapping services using data from a GIS database. OGC Web Map Service (WMS, http://opengeospatial.org/standards/wms) and Esri Map Service (http://esri.com/) are two typical web mapping service standard interfaces. In the implementation of TopoLens visualization module, we adopted the WMS standard and deployed GeoServer instances to publish web maps. The module was managed as a cloud-based service in a way that a GeoServer-based map server is encapsulated as an image on ROGER OpenStack cloud environment. Because geospatial databases are stored on the General Parallel File System (GPFS) on ROGER, each time a new map server is required, administrators can simply launch a new map server instance, and then mount the corresponding directory in the ROGER file system to the new instance to access shared geospatial database for visualization.

\subsubsection{Data Download Service}

The data download service is a simple Apache web service designed to serve result files from the shared file system using standard web protocols.

\subsubsection{Process Control}

Another challenge of the microservice architecture is ensuring all necessary services are executing normally. Rather than attempting to daemonize each service and support multiple system startup environments (i.e. init.d, upstart, system), TopoLens uses Supervisord (http://supervisord.org/), a widely-used open source package, that runs each service as a child process. In addition to starting services after a system reboot, Supervisord will monitor and automatically restart failed services. Eliminating the daemonization requirement greatly simplifies the development and testing of each service. Supervisord ensures a robust system that recovers from common system errors. 


\subsection{Big geospatial raster data preparation and sharing workflow}

The current TopoLens workflow development focuses on user-specified big geospatial raster data processing, web map based visualization, and sharing of data products and mapping services to ArcGIS Online(Figure 3). Raster data released by public authorities and government agencies are collected and organized. In addition to standard data preparation such as clipping (crop to AOI), reprojection, and resampling, pre-processing steps that are specific to the data products (e.g., calculate slope raster based on elevation data, or the reclassification of a land cover dataset) are supported. All the data processings happen in a geospatial software friendly computing environment (e.g., ROGER) where scalable geospatial algorithms are supported by HPC frameworks such as multicore CPUs, GPU, or Hadoop/MapReduce. The current workflow for visualization and sharing includes generating styled layer descriptor (SLD) for rendering raster layers, tile pyramiding for scale-dependent visualization, publishing raster data as WMS services, and sharing published map services to ArcGIS Online (Figure 3). The whole workflow is tested with the implementation on USGS 3DEP Data, which will be thoroughly discussed in the next section.

\section{CASE STUDY - USGS 3D ELEVATION PROGRAM}

TopoLens is developed as a prototype application to demonstrate a use case for the USGS 3D Elevation Program (3DEP) elevation data accessing, customizing, and sharing workflow.

\subsection{Data description and usability issues}

The 3DEP, previously known as National Elevation Dataset (NED), is one of the topographic data products produced and distributed by USGS, providing seamless raster elevation data of the conterminous United States, Alaska, Hawaii, and the island territories [18]. The elevation dataset is organized as 9361 degree by 1 degree tiles, in ArcGrid binary format, covering the continental United States (Figure 4). The total data volume for $1 / 3$-arc-second (approximately 10 meters) resolution DEM product approaches nearly $667 \mathrm{~GB}$ without compression (180 billion raster cells).

The focus of the USGS 3DEP is to provide high-quality datasets, but it does not have the resources to meet diverse end user requirements for all applications. TopoLens is a community resource to meet the most common data preparation steps, and by meeting those needs, it may have a significant impact on the large elevation data user community. These steps include the aforementioned mosaicing and clipping to an AOI, as well as reprojection to another coordinate system. The elevation datasets distributed in 3DEP use the NAD 83 coordinate system, which does not use a map projection. For research and analysis use, elevation data is often needed in projections that either preserve the area, shape, or direction properties of the real world.

In some application areas, additional processing steps need to be applied on elevation data. In hydrology, for example, extracting hydrologic features such as stream centerlines and watershed boundaries, typically requires locating and removing depressions in elevation data products as the first step. The above processing steps could all pose computational challenges if the study area is large.

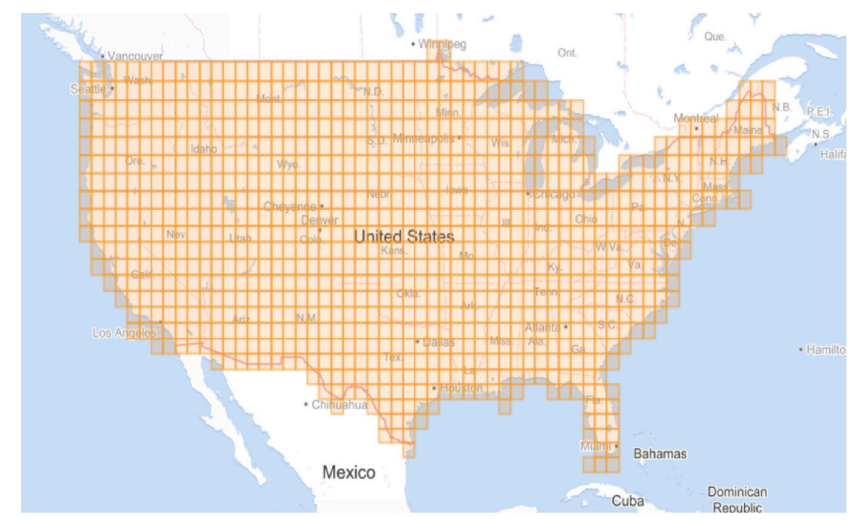

Figure 4: USGS 3DEP 1/3 arc-second resolution data coverage over continental United States.

\subsection{Elevation data preparation workflow}

The typical elevation data preparation workflow is as follows:

1) Clipping the elevation data to a geometry of area of interest. Before clipping, all 936 tiles are processed once to build a Virtual Raster (VRT), a feature of GDAL that creates a virtual dataset functioning as a mosaic of all the single tiles. Clipping is then applied on the mosaic product to generate the elevation data of the study area. Both building the VRT and clipping are performed in GDAL (with current version 1.11.4, http://gdal.org/).

2) Reprojection and resampling: The clipping output is then transformed to appropriate map projections and spatial resolution (a.k.a cell size) specified by the users. We use EPSG code (http://epsg.org/) based Coordinate Reference Systems and Coordinate Transformations for map projection specification. Four major interpolation methods, namely nearest neighbor, bilinear, cubic convolution, and majority sampling, are currently available. The implementation supports both open source reprojection and resampling tools from GDAL, and the MPI-based parallel map reprojection library pRasterBlaster [19].

3) Pre-processing for derived products. This step involves the calculation of derived products from the elevation dataset, including slope, shaded relief and pitremove. The slope product is a raster layer generated from elevation data with the slope value in degree units. The shaded relief product assigns an illumination value for each cell in the elevation raster. It is a grayscale representation of the surface in which the relative position of a hypothetical light source (e.g., the Sun) is taken into account. The pitremove product aims to locate and remove depressions in the elevation raster, which is a necessary pre-processing step for many hydrological analyses. Slope and shaded relief products are calculated in GDAL, and pitremove product is generated by TauDEM [20] with MPI support.

4) Visualization. Visualization, in this context, refers to the ability to display the requested data products in a browser application. In this step, data products need to be further processed so that they can be shared through the web as georeferenced map images, and subsequently consumed by client applications. The current TopoLens version supports WMS services publishing through GeoServer.

5) Sharing. When the visualization is ready, the sharing 


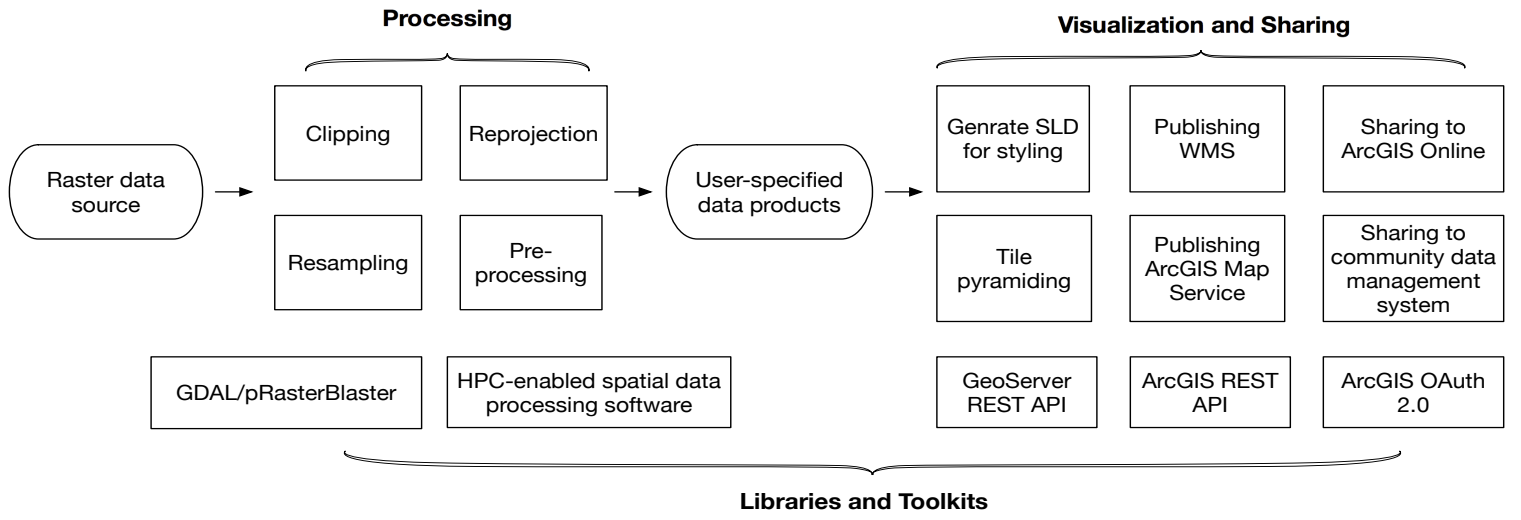

Figure 3: TopoLens data processing, visualization and sharing workflow.

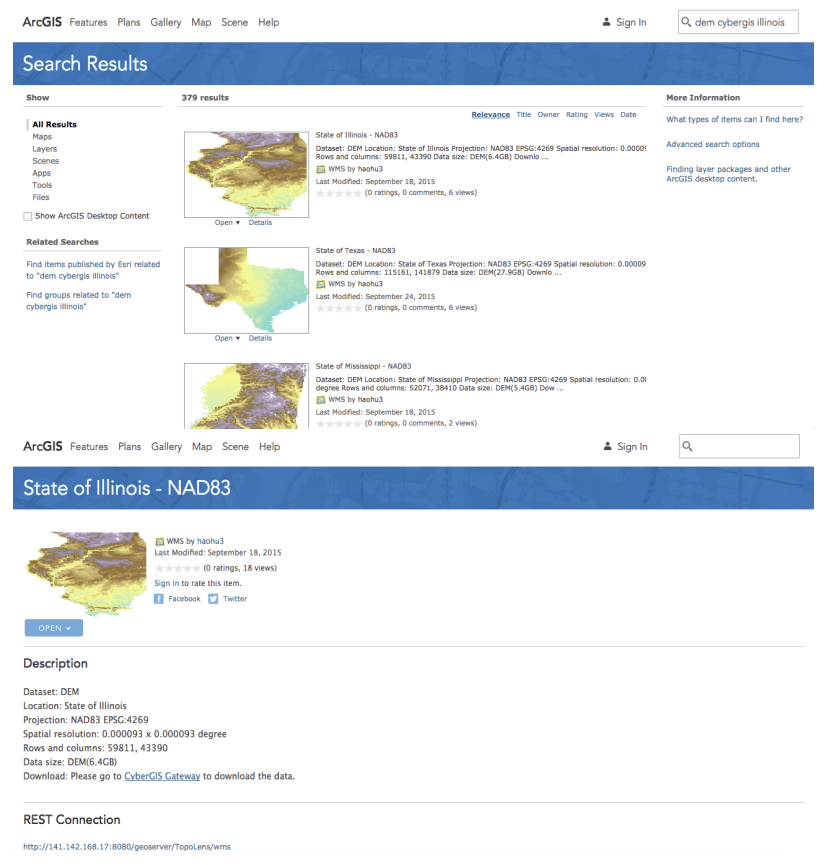

Figure 5: Sharing WMS layers of TopoLens data products to ArcGIS Online (top: ArcGIS Online TopoLens data prodcuts list, bottom: Data product details of State of Illinois NAD83 map projection $1 / 3$ arc-second resolution elevation data.)

function registers WMS layers of data products to ArcGIS Online, thus they can be searched and used as map items by ArcGIS Online users (Figure 5).

\subsection{Evaluation}

\subsubsection{Computational performance}

In the current elevation data preparation workflow, steps that require significant amounts of computation are clipping, reprojection and resampling, pre-processing for derived products, and tile pyramiding in visualization stage. The overall computational performance is determined by two major factors: the complexity of the clipping mask and the size of output raster data. The clipping mask is a poly- gon describing the geometric boundary of area of interest. The complexity of the clipping mask is represented by the number of vertices in the polygon. The output raster data size is determined by the area of the clipping mask, along with other parameters including map projection type and spatial resolution. If derived products (i.e., slope, shaded relief, and pitremove) are selected, additional computation that is proportional to the DEM raster data size is needed.

In the data preparation workflow, pRasterBlaster has been integrated as a scalable solution to improve the computational performance of reprojection and resampling by leveraging parallel computing inside of individual data processing requests with MPI (Figure 6). However, clipping capability is not currently supported in pRasterBlaster, thus requiring GDAL clipping to be done as a previous step. Figure 6 illustrates the performance of clipping, reprojection and resampling with the GDAL only and GDAL/pRasterBlaster options (from 4 to 64 processors) when requesting NAD83/Conus Albers projection in State of Illinois. When there are only 4 processors available, the GDAL-only option is more efficient than the GDAL/pRasterBlaster option. As more processors are available, the reprojection and resampling performance of the GDAL/pRasterBlaster option improves significantly, leading to better overall performance. For future work, adding clipping capabilities to pRasterBlaster to remove the additional $\mathrm{I} / \mathrm{O}$ cost posed by GDAL can further improve the performance of the data processing.

We precomputed 234 data products in 67 study areas at state level (48 contiguous states and the District of Columbia) and Hydrologic Unit Code (HUC, http://water.usgs.gov/ GIS/huc.html) regional levels (18 HUC2 regions), with 51 different map projections. The 234 computing jobs are submitted to ROGER's PBS job queue as embarrassingly parallel jobs [21]. The execution time for producing each data product ranges from 3 seconds (District of Columbia) to 12,776 seconds (Missouri Region Hydrologic Unit). The precomputed data products can be directly consumed by users for computation and visualization in other analytic workflows. The total computation took about 78 hours on ROGER's shared job queue. To improve ROGER's system utilization, we conducted a computational experiment for these 234 jobs to better understand the relationship between computing time and input data characteristics and how to leverage such information to specify the walltime of each job. Instead of submitting one job per node, we reserved 10 


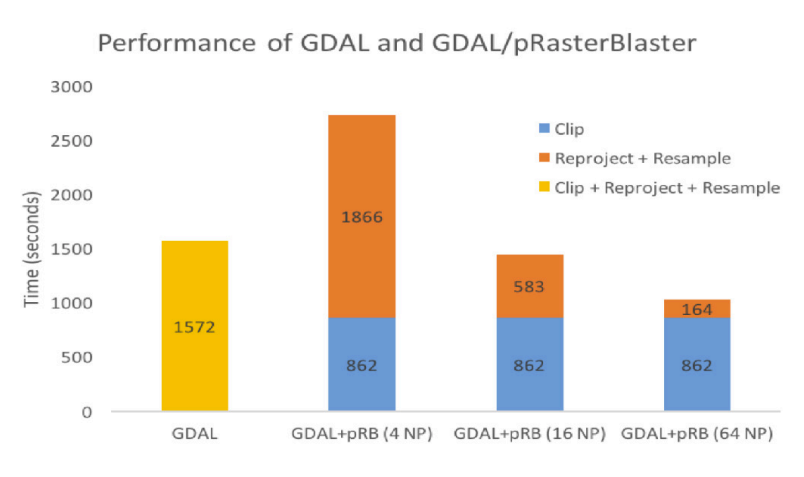

Figure 6: TopoLens performance with the GDAL only and GDAL/pRasterBlaster implementation when requesting NAD83/Conus Albers projection in State of Illinois. Horizontal axis shows the four experiments using the GDAL only and GDAL/pRasterBlaster with 4, 16, and 64 processors (NP). The performance of pRasterBlaster-based reproject and resample (in orange) shows great scalability as the number of processors increases.

ROGER nodes and evenly distributed jobs to these nodes through random shuffling. Each job uses one of the 20 cores on a ROGER node. The statistics on job completion time show that per-core scheduling reduces the total execution time significantly (Figure 7). On the other hand, it is notable that the total execution time on one node depends on the slowest job in the group. Among the 10 nodes, node $c g$-hm08 finished the last, delaying the turnaround time of this batch of jobs (Figure 7). After correlating the area of each job's spatial extent, we found that the area information can be a good indicator for developing a workflow-dependent scheduling strategy. A fast heuristic scheduling algorithm is under development to incorporate this experience for future job scheduling on ROGER, similar to the ones proposed in $[22]$.

\subsubsection{Early user experience and feedback}

TopoLens was demonstrated as a use case for 3DEP $1 / 3$ arc-second resolution elevation data accessing, customizing and sharing workflow and received positive feedback from USGS collaborators and external users. Researchers from the Illinois Prairie Research Institute and other institutions participated in a workshop held at UIUC and acted as beta testers for the application, resulting in several suggested improvements. Most of these were aimed at improving the flexibility to customize the elevation products by allowing more parameter specifications. These included additional AOI boundary file formats, additional output data formats, and optional parameters for slope and shaded relief products (such as slope type, azimuth and altitude of light source). In addition, new derived products such as flow direction and contour map were suggested for a future version. They also showed a great interest in seeing the opportunity to incorporate LiDAR data workflow in the future development.

\section{CONCLUSION AND FUTURE WORK}

Through the collaboration between researchers from UIUC
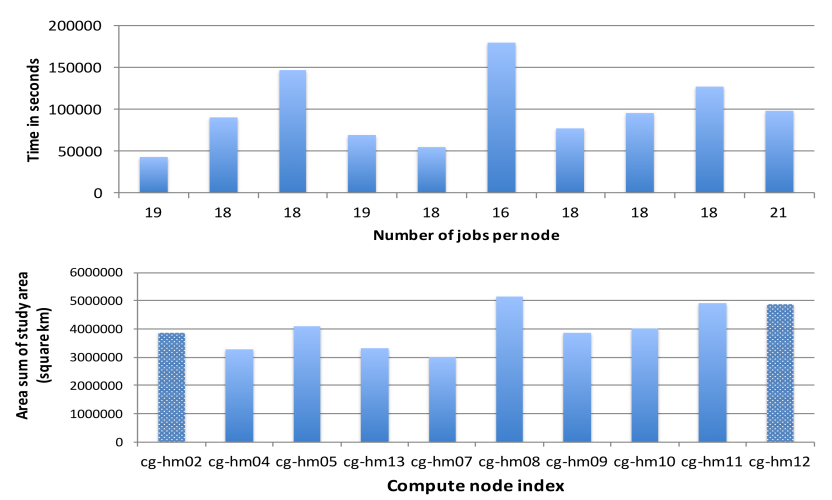

Figure 7: Relationship between the computational time and area sum of study regions allocated for each node. Similar trend is identified except for node $\mathrm{cg}$ hm02 and $c g-h m 12$, which indicates that the area of study region may be used as a measure for job scheduling.

and USGS, this study has developed a prototype application, TopoLens, as a science gateway application serving elevation raster data and map services. TopoLens demonstrates a use case for USGS 3DEP $1 / 3$ arc-second resolution elevation data accessing, customizing and sharing workflow and brings three major benefits for raster elevation data users. First, it provides a rich user interface with capabilities in elevation data searching, visualization, and parameter specifications for on-demand data requests. Secondly, high resolution elevation datasets at State and Hydrologic Unit Code (HUC) Regional levels have been precomputed. This way, they can be directly consumed by users for computation and visualization in other analytic workflows. The elimination of this step is highly beneficial to users, since elevation data at these levels are large in size, i.e., tens to hundreds of gigabytes, and are computationally expensive to process. Finally, TopoLens supports on-demand data and map services, powered by hybrid cyberinfrastructure with cloud and HPC support, to efficiently produce datasets that are customized based on user's request. As a prototype, TopoLens provides an integrated solution from web-based interface to backend cloud-based services and HPC computing environment through leveraging the hybrid design of ROGER supercomputer located at University of Illinois at Urbana-Champaign. Community users have been engaged in an early test of the application and have made helpful suggestions for future development.

Although the TopoLens prototype has received positive feedback in its current state, there are still several tasks identified for further improvement. One major enhancement is to provide detailed provenance information about the data products, including metadata defined by the original data provider, as well as the description of the methodology applied in the data preparation. Another improvement, which requires continuous effort, is to incorporate more geospatial raster data sources and workflows, e.g., OpenTopography DEMs, LiDAR datasets, and National Hydrologic Dataset (NHD) generalization workflow. To increase performance, data preparation can be more efficient through incorporating parallel solutions such as MPI-I/O based raster data 
clipping. To improve the system reliability, we are exploring an integrated solution for comprehensive HPC computing and cloud-based services monitoring and logging.

To further extend the sharing feature of TopoLens, we are currently investigating the data and service interoperability with HydroShare [9], a web portal for hydrological data and models access. We would like to register TopoLens as an external application in HydroShare, so that users can consume TopoLens REST APIs using HydroShare as the client.

\section{ACKNOWLEDGMENTS}

This work is supported in part by USGS under grant number G14AC00244, and by the National Science Foundation (NSF) under grant numbers 1047916 and 1443080. The computational work used the NSF-supported ROGER supercomputer (1429699). This work is also part of the ECSS project (award number SES090019) of XSEDE, which is supported by NSF grant number 1053575. The authors would like to acknowledge the contribution of CyberGIS Center students Dandong Yin, Liping Liu, Sunwoo Kim and Yuliya Semibratova at the University of Illinois at UrbanaChampaign.

\section{DISCLAIMER}

Any use of trade, firm, or product names is for descriptive purposes only and does not imply endorsement by the U.S. Government.

\section{REFERENCES}

[1] S. Wang, H. Hu, T. Lin, Y. Liu, A. Padmanabhan, and K. Soltani, "Cybergis for data-intensive knowledge discovery," SIGSPATIAL Special, vol. 6, no. 2, pp. 26-33, 2015.

[2] J. W. Crampton, M. Graham, A. Poorthuis, T. Shelton, M. Stephens, M. W. Wilson, and M. Zook, "Beyond the geotag: situating 'big data' and leveraging the potential of the geoweb," Cartography and geographic information science, vol. 40, no. 2, pp. 130-139, 2013.

[3] H. Chen, R. H. Chiang, and V. C. Storey, "Business intelligence and analytics: From big data to big impact.," MIS quarterly, vol. 36, no. 4, pp. 1165-1188, 2012.

[4] P. Longley, Geographic information systems and science. John Wiley \& Sons, 2005.

[5] "USGS TNM 2.0 viewer." http://viewer.nationalmap.gov/viewer/. [18 Apr 2016].

[6] L. Sugarbaker and W. Carswell, "The national map: Us geological survey fact sheet 2011-3042," 2013.

[7] S. Wang, "A cybergis framework for the synthesis of cyberinfrastructure, gis, and spatial analysis," Annals of the Association of American Geographers, vol. 100, no. 3, pp. 535-557, 2010.

[8] T. Hertel and N. Villoria, "Geoshare: Geospatial open source hosting of agriculture, resource and environmental data for discovery and decision making," 2012.

[9] D. Tarboton, R. Idaszak, J. Horsburgh, D. Ames, J. Goodall, L. Band, V. Merwade, A. Couch, J. Arrigo, R. Hooper, et al., "Hydroshare: an online, collaborative environment for the sharing of hydrologic data and models," in AGU Fall Meeting Abstracts, vol. 1, p. 1510, 2013.

[10] "ROGER user guide - ROGER - NCSA wiki." https://wiki.ncsa.illinois.edu/display/ROGER/ ROGER+User+Guide. [18 Apr 2016].

[11] "National spatial data infrastructure - federal geographic data committee." https://www.fgdc.gov/nsdi/nsdi.html. [18 Apr 2016].

[12] S. Krishnan, C. Crosby, V. Nandigam, M. Phan, C. Cowart, C. Baru, and R. Arrowsmith, "Opentopography: a services oriented architecture for community access to lidar topography," in Proceedings of the 2nd International Conference on Computing for Geospatial Research 85 Applications, p. 7, ACM, 2011.

[13] G. Mateescu, W. Gentzsch, and C. J. Ribbens, "Hybrid computing - where hpc meets grid and cloud computing," Future Generation Computer Systems, vol. 27, no. 5, pp. 440-453, 2011.

[14] J. Qiu, J. Ekanayake, T. Gunarathne, J. Choi, S.-H. Bae, H. Li, B. Zhang, T.-L. Wu, Y. Ruan, S. Ekanayake, et al., "Hybrid cloud and cluster computing paradigms for life science applications," BMC bioinformatics, vol. 11, no. 12, p. 1, 2010.

[15] C. Yang, M. Goodchild, Q. Huang, D. Nebert, R. Raskin, Y. Xu, M. Bambacus, and D. Fay, "Spatial cloud computing: how can the geospatial sciences use and help shape cloud computing?," International Journal of Digital Earth, vol. 4, no. 4, pp. 305-329, 2011.

[16] K. Evangelidis, K. Ntouros, S. Makridis, and C. Papatheodorou, "Geospatial services in the cloud," Computers $\&$ Geosciences, vol. 63, pp. 116-122, 2014.

[17] Y. Liu, A. Padmanabhan, and S. Wang, "Cybergis gateway for enabling data-rich geospatial research and education," Concurrency and Computation: Practice and Experience, vol. 27, no. 2, pp. 395-407, 2015.

[18] "The national map: Elevation." http://nationalmap.gov/elevation.html. [18 Apr 2016].

[19] M. Finn, Y. Liu, D. Mattli, Q. Guan, K. Yamamoto, E. Shook, and B. Behzad, "prasterblaster: High-performance small-scale raster map projection transformation using the extreme science and engineering discovery environment," in Abstract presented at the XXII International Society for Photogrammetry $\&$ Remote Sensing Congress, Melbourne, Australia, 2012.

[20] D. G. Tarboton, "Terrain analysis using digital elevation models (taudem)," Utah Water, 2005.

[21] M. D. McCool, A. D. Robison, and J. Reinders, Structured parallel programming: patterns for efficient computation. Elsevier, 2012.

[22] T. D. Braun, H. J. Siegel, N. Beck, L. L. Bölöni, M. Maheswaran, A. I. Reuther, J. P. Robertson, M. D. Theys, B. Yao, D. Hensgen, et al., "A comparison of eleven static heuristics for mapping a class of independent tasks onto heterogeneous distributed computing systems," Journal of Parallel and Distributed computing, vol. 61, no. 6, pp. 810-837, 2001. 